\title{
Multimodal Background Modeling Using RGB-Depth Features
}

\author{
Rim Trabelsi ${ }^{1,3}$, Fethi Smach ${ }^{2}$, Issam Jabri ${ }^{1}$, and Ammar Bouallegue ${ }^{3}$ \\ 1 IResCoMath Unit, National Engineering School of Gabes, University of Gabes \\ rim.trabelsi@ymail.com, \\ issam.jabri@enig.rnu.tn \\ 2 Profil Technology, 49 Rue de la Vanne, 92120 Montrouge, France \\ fethi.smach@gmail.com \\ 3 SysCom Laboratory, National Engineering School of Tunis, \\ University of Tunis El Manar \\ ammar.bouallegue@enit.rnu.tn
}

\begin{abstract}
This paper presents a method of background subtraction that uses multimodal information, specifically depth and appearance cues, to robustly separate the foreground in dynamic indoor scenes. To this end, RGB-Depth data from a Microsoft Kinect sensor are exploited. We propose an extension of one from the most effective technique for background modeling in real time: Kernel Density Estimation with Fast Gauss Transform technique. Experimental results show that our proposed deals well with gradual/sudden illumination changes, shadows and dynamic backgrounds.
\end{abstract}

Keywords: RGB-Depth, Multimodal Background subtraction, Kernel Density Estimation, Fast Gauss Transform.

\section{Introduction}

Many applications in computer vision begin with a step of detecting change. The objective is usually double. Firstly, it simplifies subsequent treatments by locating regions of interest in the image to optimize the computational time. Then, the number of false detections is also reduced because the regions of the image where the probability of finding an object is very low are eliminated. This is a crucial phase because the following steps will be based on this result. From a model of the environment and an observation or a series of successive observations, we try to detect what has changed. There is variety of methods for detecting change, the most known is the background subtraction. This technique consists of subtracting the observed image from an estimated image and thresholding the result to generate the objects of interest. This task is very challenging in the case of real scenes with dynamic background. In fact, there are many causes related to the nature of the filmed scene that can affect the quality of background subtraction. Among these difficulties we can mention: gradual/sudden illumination changes, shadows casted by foreground objects, animated textures 
in the background (waving trees, television or computer display screens, etc.), introduction or removal of static objects in the scene, etc.

Many models of the background have been presented in the literature to handle these difficulties. Stauffer and Grimson [1] represented each pixel in the RGB color space by a mixture of K Gaussian distributions. This approach relatively takes into account multimodal background. However, the number of Gaussians must be pre-fixed with a large number of parameters to determine empirically and this requires a great effort to fix the optimal combination for a given situation. Many others use a median-based approach. For example, Lo and Velastin [2] proposed to use the median value of the last $\mathrm{N}$ frames as the background model. This manner is very fast but it can't cope with multiple modal background distributions. Oliver et al. 3] proposed to perform a principal component analysis to construct a background model based on an eigenvalue decomposition (commonly called eigen-background). This procedure can be subject to variations improving its efficiency. However, it isn't explicitly specified what images should be part of the initial sample, and whether and how such a model should be updated over time. Another method presented by Kim et al. [4, based on an encoding table (codebook) to handle multimodal backgrounds. From a training sequence, the method assigns each pixel of the background more sets of key values (codewords) describing all the colors of a pixel background can take.Kernel density estimation, proposed by Elgammal et al. [5], is one of the most popular non-parametric techniques to model densities. It provides a flexible framework to represent multi-modal densities. However, its very high memory requirements and computational complexity inhibit the use of this method in real-time applications. All these methods suffer from either the lack of flexibility, by fixing or limiting or large memory requirement. These problems are aggravated in real-time computer vision applications since background model required to be updated as new data becomes available. Nevertheless, most of attempts which have tried to reuse these approaches to deal with several problems in realistic environment were based on the use of monoscopic cues.

Recently, 3D data acquisition techniques are in full swing. The postponement of such measures on an image is referred to as depth map, which is invariant to illumination changes, shadows and reflections. Some works used to benefit from 3D data by using passive sensors mainly stereoscopic ones to embitter background modeling. Ivanov et al. [6] proposed the use of simplified stereo algorithm to perform the segmentation, which requires two camera views to be available. The background disparity is computed off-line. Then for every frame, it is used to wrap every right image on the left one to measure similarity. Yet, the quality of estimated disparity map wasn't quite accurate. Liu and Wang [7] exploited an advanced technique for computing disparity. They propose an hierarchical approach for background subtraction built in two views respectively with discrete wavelet transformation. Alahi et al. [8] suggested an algorithm to extract foreground silhouettes to produce a dense disparity based on a Total Variation framework 9. In this paper, we present a fast and robust method for background modeling, updating and subtraction in a dynamic indoor 
environment based on a RGB-Depth data. We propose an extension to Kernel Density Estimation (KDE) [5] which has proven a great efficiency to deal with problem of multimodality but it suffer from computational cost. To solve this, we resort to the Fast Gauss Transform (FGT) which had successfully accelerated the KDE [10]. This technique is employed in our case to model the background using both color and depth data.

This paper is organized as follows : in section 2, we highlight the benefits of the multimodal information mainly color and depth information to enhance background and foreground segmentation. In section 3, the different phases of the proposed method are described. Finally, experimental results are shown in section 4 .

\section{Benefits of RGB-D Data for Background Subtraction}

As mentioned in section 11, background and foreground detection have been actively studied for decades and a variety of approaches have been proposed. However, many issues still unresolved and challenging until now like scenes with dynamic background, uncontrolled illumination and shadows. While traditional approaches rely on $2 \mathrm{D}$ data and their features, the development of $3 \mathrm{D}$ cameras and depth sensors created new opportunities to advance the state-of-the art in this field. In fact, depth information is less affected by those challenging issue such as shadows and animated textures. Yet, the extraction of depth may be itself affected by the illumination changes. For this reason, the fusion between multimodal information is very required to achieve a robust segmentation. On these days, depth information can be extracted in real-time using several technologies like stereovision techniques using passives sensors [11, TOF (Time Of Flight) cameras [12] and the Microsoft Kinect sensor [13], among others. In this work, our technique exploit the new 3D emergent sensor from Microsoft Kinect for Windows which provides depth maps with good quality in real time and simultaneously with the RGB stream. Previous attempts which have used both 2D and 3D data for background subtraction have used TOF [14 or passive sensors [15] improve efficiency in many difficult conditions but their results is not as excepted due to the poor quality of their depth maps under complex indoor scenes.

\section{Proposed Technique}

The designed method described in Figure 1includes mainly five phases. We start firstly by prepocessing both depth an color sequences to reduce inevitably noise. Then, we store the first $\mathrm{N}$ frames (usually 10 frames) to build the background model. To this end, we opt to an extension of the KDE/FGT technique to estimate the background. This technique will be developed in section 3.1 and 3.2. Afterwards, we subtract the background and this lead to the segmentation of the foreground. After each frame, we update the background to adapt changes. Here, we pass a mask where pixels with true value will be masked out of the update. 


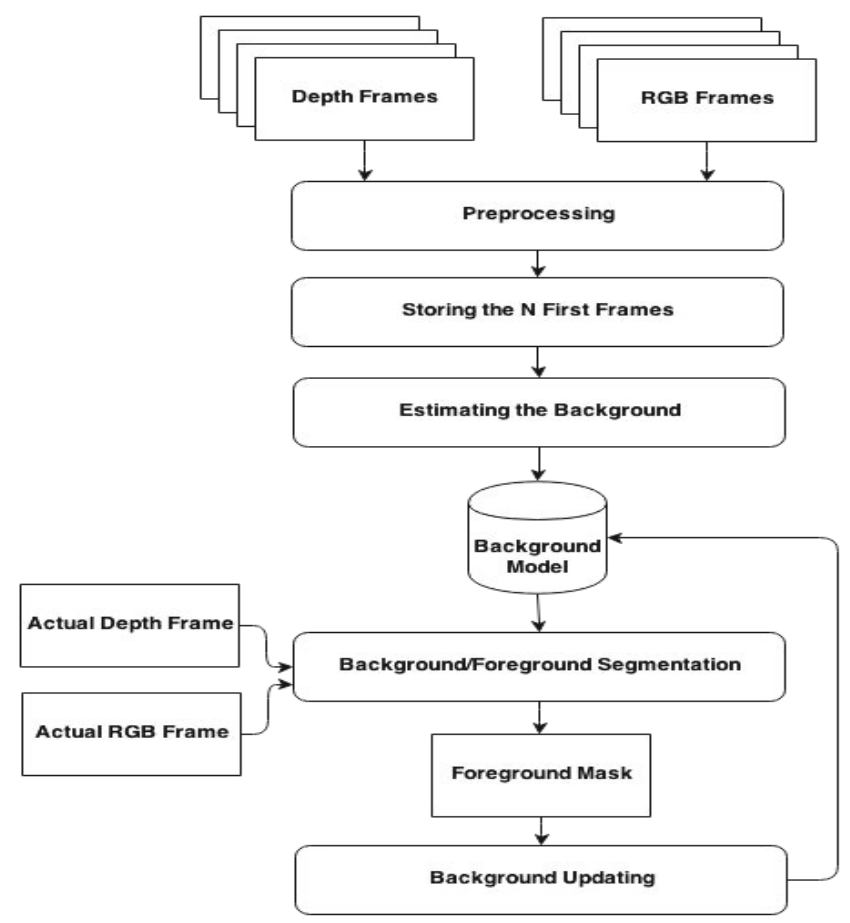

Fig. 1. Overall view of the designed method

\subsection{Kernel Density Estimation for Depth-Appearance Modeling}

Kernel Density Estimation (KDE) is a non-parametric method used mainly in statistic 16 but also in computer vision researches [17. Elgammal et al. in [18. have proposed to employ this technique to model the background color distribution. The density estimation of the background is computed using the last n observations. Assume $x_{i}, i=1, \ldots, n$, be scalar measurements drawn from an arbitrary probability distribution $f(x)$. The kernel density estimate of this distribution $\hat{f}(x)$ is obtained based on a kernel function $K(u)$ and a bandwidth $h$ as

$$
\hat{f}(x)=\frac{1}{n h} \sum_{i=1}^{n} K\left(\frac{x-x_{i}}{h}\right)
$$

The kernel functions considered here satisfy the following properties

$$
\begin{aligned}
& K(u)=K(-u) \geq 0 \\
& K(0) \geq K(u) \text { for } u \neq 0 \\
& K(u)=0 \text { for }|u|>0 \\
& \int_{-1}^{1} K(u)=1
\end{aligned}
$$

As mentioned above, in this work we propose to use depth and the RGB data to model the background with KDE technique. For this purpose, we choose 
to model the background at every time $t$ and for every pixel $p$ by a vector $X_{(t, p)}=<D_{(t, p)}, C_{(t, p)}>$ which $C(t, p)=R^{2}(t, p)+G^{2}(t, p)+B^{2}(t, p)$ is the average value of each color component and $D(t, p)$ is the depth value. We can estimate the density function at any point using the product of one dimensional kernel as

$$
\hat{f}(C, D)=\frac{1}{n h_{C} h_{D}} \sum_{i=1}^{n} K\left(\frac{C-C_{i}}{h_{C}}\right) K\left(\frac{D-D_{i}}{h_{D}}\right)
$$

Using Gaussian kernels with different bandwidths in each dimension, the density estimation can be evaluated as a sum of Gaussians as

$$
\hat{f}(C, D)=\frac{1}{n h_{C} h_{D}} \sum_{i=1}^{n}\left(\frac{1}{\sqrt{2 \pi h_{C}^{2}}} e^{-\frac{\left(C-C_{i}\right)^{2}}{2 h_{C}^{2}}}\right)\left(\frac{1}{\sqrt{2 \pi h_{D}^{2}}} e^{-\frac{\left(D-D_{i}\right)^{2}}{2 h_{D}^{2}}}\right)
$$

Where $h_{C}$ and $h_{D}$ represent respectively the bandwidths of color and depth space dimension. Usually, the color variable has more variance than the depth variable and therefore wider kernels should be used in the color dimension. One major issue that needs to be addressed when using kernel density estimation technique is the choice of suitable kernel bandwidth (scale).

The major drawback of KDE techniques is their computational cost. The use of such an approach requires a way to efficiently evaluate the estimate $\hat{f}(x)$ at any new target point $x$. In general, given $N$ original data samples and $M$ target points at which the density need to be evaluated, the complexity is $\mathrm{O}(\mathrm{NM})$ evaluations of the kernel function, multiplications and additions. This is drawback makes unattainable real-time aspect in many applications. To reduce computational time, Fast Gauss Transform (FGT) [5] are used to reduce complexity with KDE.

\subsection{Fast Gauss Transform}

The fast Gauss transform proposed by Greengard and Strain [10] reduces the computational complexity of the evaluation of the sum of $N$ Gaussians at $M$ points in $d$ dimensions from $O(M N)$ to $O(M+N)$.

$$
G\left(y_{i}\right)=\sum_{i=1}^{N} q_{i} e^{-\left\|y_{i}-x_{i}\right\|^{2} / h^{2}}, q_{i} \in \mathbb{R}, x_{i} \in \mathbb{R}^{d}
$$

where $q_{i}$ are the weight coefficients, "source" points $\left\{x_{i}\right\}_{i=1, \ldots, N}$ are the centers of the Gaussians, $h$ is the bandwidth parameter of the Gaussians. The sum of the Gaussians is evaluated at each of the "target" points $\left\{y_{j}\right\}_{j=1, \ldots, M}$. The final algorithm is of complexity $O(N+M)$ instead of $O(N M)$ by direct evaluation.

A generalization of the original algorithm is needed to handle the case where the bandwidth is different in each dimension:

$$
G\left(y_{i}\right)=\sum_{i=1}^{N} q_{i} e^{-\sum_{j=1}^{d}\left(\frac{\left(y_{i}-x_{i}\right)_{j}}{h_{j}}\right)^{2}}
$$


Since the variation of depth and color are different, bandwidths of kernels for both of them are also different, then

$$
G\left(y_{i}\right)=\sum_{i=1}^{N} q_{i} e^{-\left(\frac{\left(y_{i}-x_{i}\right)_{C}}{h_{C}}\right)^{2}-\left(\frac{\left(y_{i}-x_{i}\right)_{D}}{h_{D}}\right)^{2}}
$$

At each new frame, it is desired to estimate the RGB-D density $\hat{f}_{x}(C, D)$ corresponding to each pixel $x$, at each pixel in the foreground. The FGT algorithm is used to efficiently compute these probabilities wherein sources are the sample $V=\left\{X_{i}=\left(C_{i}, D_{i}\right)\right\}$ while the targets are the vectors $\langle C, D\rangle$ at each evaluation location. Since both the sources and the targets are clustered in the same spaces, the FGT algorithm gives a significant speedup. The estimation of the bandwidth for each dimension is done off-line.

\section{Experiments}

In this section, we tested the proposed approach on a variety of indoor sequences which are taken from an RGB-D People Dataset [19] 20. This dataset contains RGB-D frames acquired in a university hall from three vertically mounted Microsoft Kinect sensors. To highlight the efficiency of the use of both color and depth cues, we have chosen three challenging sequences as shown in Figure 2.

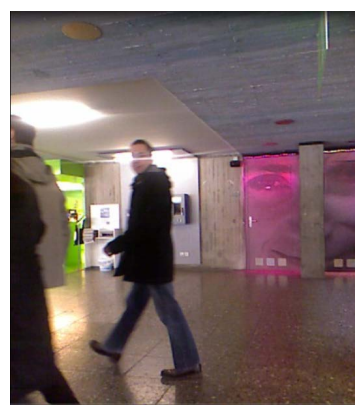

(a) Frame from sequence $n^{\circ} 01$

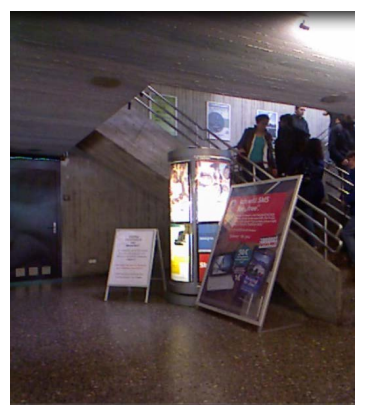

(b) Frame from sequence $n^{\circ} 02$

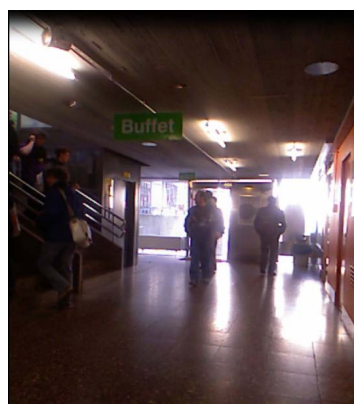

(c) Frame from sequence $n^{\circ} 03$

Fig. 2. Video sequences used for our experiments

In the first sequence, as shown in Figure 2(a), people walk in and out of the camera field and doors are opened and closed at odd times and causes local and sudden illumination changes. Figure 2,(b) shows a sample frame from the second sequence in which we have furthermore a dynamic background due to the presence of rotating display screen. In third test sequence (Figure 2.(c)), there were moving many persons in the scene during initialization and the dark floor with the big amount of the uncontrolled illumination complicated the detection. 
The same bandwidths were used for all the sequences. In those cases the RGB-Depth based method generated foregrounds representing the changes and it responds entirely to the illumination changes and dynamic background as anticipated (Please see Table 1). However, inaccuracies in the depth maps lead to some false negatives and undetected foreground regions with similar depth to the background and some false positives caused by noise of color frames.

Table 1. Original frames, depth frames, and Foreground/Background masks obtained in the test sequence

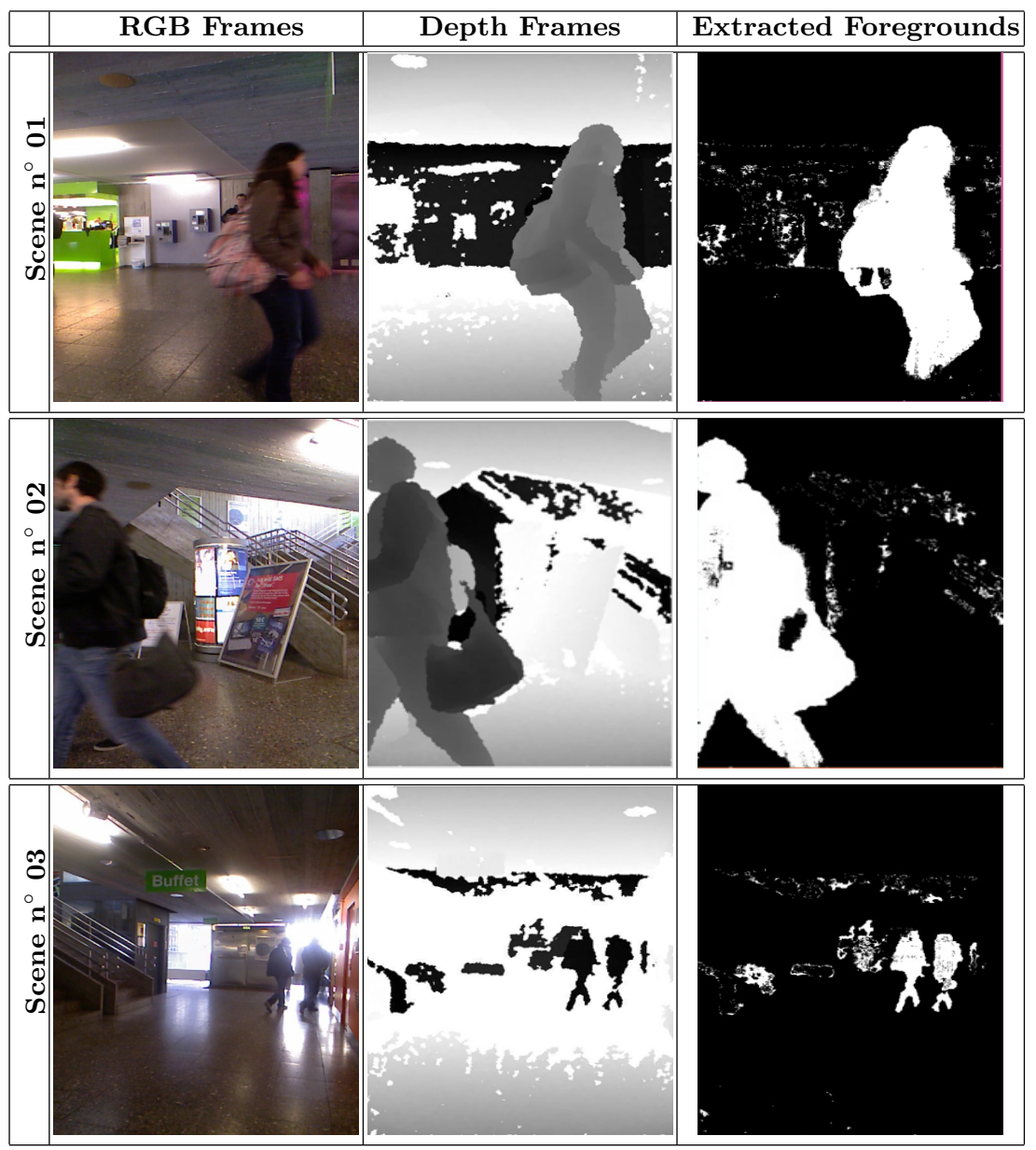




\section{Conclusion}

The key to our approach is to improve the quality of the foreground and background segmentation by means of the combination of RGB and depth cues extracted by Microsoft Kinect sensor. We have proposed an extension of the Kernel density estimation proposed by Elgammal et al [5]. Experimental studies are conducted to illustrate the validity of the proposed approach in complex indoor scenes. It responds entirely to the constraints of the uncontrolled illumination and dynamic backgrounds. Quantitative validation for this work is not feasible because the literature lack of a dataset containing color and depth frames issued from Kinect sensor and their corresponding backgrounds/foregrounds ground truth. The future work will include the study of computational time and the gain of the implemented FGT technique as well as a set of data including RGB$\mathrm{D}$ data with ground truth.

\section{References}

1. Stauffer, C., Grimson, W.E.L.: Adaptive background mixture models for real-time tracking. In: IEEE Conference on Computer Vision and Pattern Recognition, vol. 2 (1999)

2. Lo, B., Velastin, S.: Automatic congestion detection system for underground platforms. In: IEEE International Symposium on Intelligent Multimedia, Video and Speech Processing, pp. 158-161 (2001)

3. Oliver, N.M., Rosario, B., Pentland, A.P.: A bayesian computer vision system for modeling human interactions. IEEE Transactions on Pattern Analysis and Machine Intelligence 22(8), 831-843 (2000)

4. Kim, K., Chalidabhongse, T.H., Harwood, D., Davis, L.: Real-time foregroundbackground segmentation using codebook model. Real-time Imaging 11(3), 172$185(2005)$

5. Elgammal, A., Duraiswami, R., Davis, L.S.: Efficient kernel density estimation using the fast gauss transform with applications to color modeling and tracking. IEEE Transactions on Pattern Analysis and Machine Intelligence 25(11), 14991504 (2003)

6. Ivanov, Y., Bobick, A., Liu, J.: Fast lighting independent background subtraction. International Journal of Computer Vision 37(2), 199-207 (2000)

7. Liu, T., Wang, G.: A hierarchical approach for robust background subtraction based on two views. In: WRI Global Congress on Intelligent Systems, vol. 4, pp. 325-329. IEEE (2009)

8. Alahi, A., Bagnato, L., Matti, D., Vandergheynst, P.: Foreground silhouette extraction robust to sudden changes of background appearance. In: 19th IEEE International Conference on Image Processing (ICIP), pp. 1229-1232. IEEE (2012)

9. Bagnato, L., Frossard, P., Vandergheynst, P.: A variational framework for structure from motion in omnidirectional image sequences. Journal of Mathematical Imaging and Vision 41(3), 182-193 (2011)

10. Greengard, L., Strain, J.: The fast gauss transform. SIAM Journal on Scientific and Statistical Computing 12(1), 79-94 (1991) 
11. Trabelsi, R., Smach, F., Jabri, I., Abdelkefi, F., Snoussi, H., Bouallegue, A.: An endeavour to detect persons using stereo cues. In: Zaman, H.B., Robinson, P., Olivier, P., Shih, T.K., Velastin, S. (eds.) IVIC 2013. LNCS, vol. 8237, pp. 358370. Springer, Heidelberg (2013)

12. Shim, H., Lee, S.: Hybrid exposure for depth imaging of a time-of-flight depth sensor. Opt. Express 22(11), 13393-13402 (2014)

13. Shotton, J., Sharp, T., Kipman, A., Fitzgibbon, A., Finocchio, M., Blake, A., Cook, M., Moore, R.: Real-time human pose recognition in parts from single depth images. Communications of the ACM 56(1), 116-124 (2013)

14. Schiller, I., Koch, R.: Improved video segmentation by adaptive combination of depth keying and mixture-of-gaussians. In: Heyden, A., Kahl, F. (eds.) SCIA 2011. LNCS, vol. 6688, pp. 59-68. Springer, Heidelberg (2011)

15. Fernandez-Sanchez, E., Rubio, L., Diaz, J., Ros, E.: Background subtraction model based on color and depth cues. Machine Vision and Applications 25(5), 1211-1225 (2014)

16. Wand, M.P., Jones, M.C.: Kernel smoothing, vol. 60. CRC Press (1994)

17. Chen, H., Meer, P.: Robust computer vision through kernel density estimation. In: Heyden, A., Sparr, G., Nielsen, M., Johansen, P. (eds.) ECCV 2002, Part I. LNCS, vol. 2350, pp. 236-250. Springer, Heidelberg (2002)

18. Elgammal, A., Harwood, D., Davis, L.: Non-parametric model for background subtraction. In: Vernon, D. (ed.) ECCV 2000. LNCS, vol. 1843, pp. 751-767. Springer, Heidelberg (2000)

19. Spinello, L., Arras, K.O.: People detection in RGB-D data. In: IEEE/RSJ International Conference on Intelligent Robots and Systems (IROS), pp. 3838-3843 (2011)

20. Luber, M., Spinello, L., Arras, K.O.: People tracking in RGB-D data with online boosted target models. In: IEEE/RSJ International Conference on Intelligent Robots and Systems (IROS), pp. 3844-3849 (2011) 